\title{
MARKETING DE RELACIONES: UN ANÁLISIS DE SU DEFINICIÓN
}

\author{
SARMIENTO GUEDE José Ramón ${ }^{1}$ \\ ESERP Business School \\ prof.jrsarmiento@eserp.com \\ Universidad Internacional de la Rioja \\ joseramon.sarmiento@unir.net
}

Material original autorizado para su primera publicación en la revista académica REDMARKA. Revista Digital de Marketing Aplicado.

https://doi.org/10.17979/redma.2016.01.016.4871

Recibido: 31 Diciembre 2015

Aceptado: 11 Mayo 2016

\section{Resumen}

La mayor dificultad para definir el Marketing de Relaciones radica principalmente en que el concepto denota un conjunto de actividades que

${ }^{1}$ Profesor contratado de ESERP Business School. Profesor colaborador de la Universidad Internacional de la Rioja. Doctor de en Marketing por la Universidad Rey Juan Carlos. Autor, entre otras publicaciones del libro el Marketing de Relaciones: una aproximación a las relaciones virtuales. De artículos como "la comunicación de boca en boca vs. la de boca en boca electrónica: análisis de sus principales diferencias" o "el impacto de los medios sociales en la estructura del sistema de distribución turístico: análisis y clasificación de los nuevos proveedores de servicios turísticos en el entorno online". 
varían según el contexto. Por ello, hay autores como Dann y Dann que sostienen que, para definir y obtener una idea clara del Marketing de Relaciones, bastaría con recurrir a cuatro definiciones que se realizan sobre el término y a cinco variables que se desarrollan sobre su proceso. En consecuencia, una investigación como la que proponemos está más que justificada, marcándonos un único objetivo, que es proporcionar unas características que definan el Marketing de Relaciones. Para alcanzar a dicho objetivo, conviene, pues, empezar por analizar y revisar la literatura existente sobre dicho concepto.

Palabras clave: Definición, Marketing de Relaciones, CRM, Marketing Relacional

\begin{abstract}
The greatest difficulty to define relationship marketing lies mainly in the concept denotes a set of activities that vary according to context. Therefore, some authors like Dann and Dann who argue that, to define and get a clear idea of relationship marketing, suffice to use four definitions that are made on the term and five variables that develop on their process. Accordingly, an investigation as performed is more than justified, marking us one goal, which is to provide features that define the relationship marketing. To reach this goal we will use a literature review.
\end{abstract}

Keywords: Definition, Relationship Marketing, CRM, Relational Marketing

\title{
1. INTRODUCCIÓN
}


Durante los últimos años, el Marketing de Relaciones se ha utilizado para reflejar y cubrir conceptualmente gran variedad de temas y perspectivas (Nevin, 1995). En consecuencia, por Marketing de Relaciones no puede entenderse un concepto totalmente unitario con unos objetivos y unas estrategias bien definidas; más bien, parece que por esta denominación ha de entenderse una "filosofía de paraguas" (Egan, 2011:32) con numerosas variaciones. En la práctica, es la filosofía que han estado aplicando los pequeños comerciantes durante toda la vida. Pues, aunque carecían de la tecnología con la que ahora contamos, "los antiguos comerciantes, hoy conocidos como tenderos, al igual que las actuales empresas manejadas por sus mismos dueños, llevaban la relación con sus clientes más fieles de una manera tan personal que los conocían y los mencionaban por su nombre, sabían de sus gustos, les trataban de una forma que a ellos les satisficiera, logrando a cambio su lealtad" (Publicidad y Mercadeo, 1994:66; en Barroso y Martín, 1999).

Uno de los primeros trabajos de investigación sobre el concepto del Marketing de Relaciones fue el de Harker (1999), trabajo en el que estudió y compiló 28 definiciones. Un año más tarde, Dann y Dann (2000), revisando la literatura del Marketing de Relaciones, llegaron a constatar que habían sido publicadas cerca de 50 definiciones. $Y$ en el último trabajo de investigación sobre la definición, publicado por Agariya y Singh (2011), ya se analizaron 72 definiciones. A este gran número de definiciones, la investigación podría añadir la "utilización de otros términos, bien como sustitutos del Marketing de Relaciones, o bien como descriptores de conceptos similares" (Buttle, 1996:2). Nos referimos a conceptualizaciones como las siguientes: "marketing directo", "marketing de base de datos", "micro-marketing", "marketing one-to-one", "marketing de fidelizacion", "marketing simbiótico", "marketing interactivo", "marketing segmentado", "marketing envolvente", "asociación de clientes", 
"marketing individual", "marketing relevante", "marketing de unión", "marketing de frecuencia", "marketing integrado" y "marketing de dialogo". Muchas de estas variantes describen un aspecto de las relaciones o están estrechamente ligadas a la filosofía del Marketing de Relaciones.

El "Marketing Directo" o el "Marketing de Base de Datos" son conceptos generalmente asociados, por lo que en algunas ocasiones pueden solaparse con el de Marketing de Relaciones. Pues, aunque estos conceptos no reflejan plenamente el Marketing de Relaciones, puede afirmarse que comparten algunas estrategias y tácticas relacionales fácilmente reconocibles. Así, el Marketing Directo se define en términos de "conectar de manera directa con consumidores metacuidadosamente seleccionados, ya sean segmentados o individualizados, a menudo sobre una base interactiva y personal" (Kotler y Armstrong, 2013:422). Y, por Marketing de Base de Datos, se viene entendiendo "recoger información exhaustiva y organizada sobre clientes individuales o prospectos que incluyen datos geográficos, demográficos y conductuales" (Kotler y Armstrong, 2013:422). Sin embargo, hemos de concluir que el "Marketing Directo" y el "Database Marketing" contienen características mucho mas transaccionales que relacionales (Egan, 2011:33).

Otro concepto que intentamos diferenciar del de "Marketing de Relaciones" es el de "Customer Relationship Managment" (CRM). Así, Payne define el "CRM" como "un enfoque empresarial que busca crear, desarrollar y mejorar las relaciones con un cliente, en concreto con la finalidad de mejorar el valor, la rentabilidad de las empresas y, por lo tanto, maximizar el valor de los accionistas" (2006:4). Y, aunque el CRM a menudo se asocia con el Marketing de Relaciones, el termino en sí mismo surgió "de las TIC utilizadas por la comunidad de los proveedores" a mediados de los 90 (Payne y Frow, 2005:167). En realidad, el CRM es un herramienta utilizada para compilar los 
datos y actividades que se desarrollan en torno al binomio relacional clienteproveedor. Según Payne (2006), el CRM aúna el potencial de las nuevas tecnologías con el nuevo pensamiento de Marketing para ganar rentabilidad en las relaciones a largo plazo.

Para definir el Marketing de Relaciones, la mayor dificultad radica principalmente en que el concepto denota un conjunto de actividades que varían según el contexto. Incluso hay algunos autores que llegaron a afirmar que el Marketing de Relaciones se ha convertido en una "palabra de moda" (Nevin, 1995:502); o que se ha cargado de ambigüedad dentro de la literatura del Marketing (Parvatiyar y Sheth, 2000). Las razones que llevan a la divergencia existente entre lo que debería o no debería constituir el Marketing de Relaciones pueden ser reducidas dos (Harker, 1999): (1) La primera de ellas surge como consecuencia de una perspectiva emergente por la que el Marketing de Relaciones se ve como un concepto relativamente joven a pesar de haber ejercido durante los últimos años una gran influencia sobre la disciplina del Marketing y sobre el ámbito empresarial. Por ello, se aconseja esperar a que este paradigma del Marketing esté completamente asentado. (2) Y la segunda de ellas es consecuencia directa de la variedad de las contribuciones implicadas en su desarrollo. Sabemos que los antecedentes académicos, como el Marketing de Servicios o como el Marketing Industrial, han tenido una gran trascendencia a la hora de la conceptualización.

\section{METODOLOGÍA, ESTRUCTURA Y OBJETIVOS}

El presente trabajo de revisión bibliográfica trata de proporcionar de una forma detallada, selectiva y crítica toda la información publicada sobre el concepto del Marketing de Relaciones. La revisión se puede reconocer como un estudio en sí mismo, en el cual el revisor se formula un interrogante, recoge 
datos (en la forma de artículos previos), los analiza y extrae una conclusión. La diferencia fundamental entre una revisión y un trabajo original o estudio primario, es la unidad de análisis, no los principios científicos que se aplican.

En consecuencia, el trabajo lo hemos estructurado en dos partes: (a) en la primera, presentaremos el marco teórico que circunscribe este trabajo de investigación, esto es, la definición nominal y conceptual del Marketing de Relaciones; (b) en la segunda parte se presentan las principales conclusiones.

Como afirma Harker, es necesario llegar a una unificación conceptual del Marketing de Relaciones a fin de poder hablar el mismo lenguaje. En caso contrario, se hace muy difícil dar respuesta cumplida a la pregunta fundamental: ¿qué es el Marketing de Relaciones? En consecuencia, una investigación como la realizada está más que justificada, marcándonos un único objetivo, que es proporcionar unas características que definan el Marketing de Relaciones.

\section{MARCO TEÓRICO}

El marco teórico de este trabajo de investigación circunscribe la definición nominal y conceptual del Marketing de Relaciones

\subsection{DEFINICIÓN NOMINAL}

El término "relation" es un sustantivo abstracto cuyo significado es "the way in which two or more concepts, objects, or people are connected; a thing's effect on or relevance to another = la manera en la que dos o más conceptos, objetos o personas están conectadas; el efecto o relevancia de una cosa respecto de otra" (Oxford Spanish Dictionary, 2009). Y la traducción literal al 
español suele hacerse con el sustantivo "relación" que significa conexión, correspondencia de algo con otra cosa, mientras que el efecto de algo lo traduce el adjetivo "relacional": perteneciente o relativo a la relación (véase Tabla 1). Al añadir el sufijo -ship a la palabra inglesa "relation", se forma un nuevo sustantivo abstracto que se refiere a una relación entre dos o más personas y cosas; y significa "being related: categoría o estado de conexión". En este caso, la traducción correcta de "Relationship Marketing" en español es "Marketing de Relacion" (singular) o "Marketing de Relaciones" (plural) en donde el sustantivo "relación" está tomado en su sentido genérico, o de clase. Quiere decir que es de relaciones y no de negocios ni de cualquier otra cosa.

Como se puede observar en la tabla 1, la utilización del término "marketing relacional" en tanto traducción literal de "Relationship Marketing" es incorrecta, porque el adjetivo "relational" en inglés, igual que "relacional" en español, significa "perteneciente o relativo a la relación", pero no significa "la manera o estado de estar relacionado", concepto sustantivo que en inglés recoge el abstracto "relationship" y en español cubre el sustantivo genérico sin artículo, ya sea en singular, ya en plural: Marketing de Relación o Marketing de Relaciones. Por tanto, las traducciones como "marketing relacional" se entienden en español literalmente como "marketing relativo a la relación", cuando debería de entenderse "Marketing de Relaciones", denotación de estar relacionado o de aplicación del Marketing.

Tabla 1: Traducción correcta de "Relationship Marketing".

"Relationship Marketing" (sustantivo)
"Relational marketing" (Adjetivo) $\quad\left\{\begin{array}{l}\text { Marketing de Relación (singular) } \\ \text { Marketing de Relaciones (plural) } \\ \text { Marketing relacional (Adjetivo) }\end{array}\right.$

Fuente: Elaboración propia

\subsection{DEFINICIÓN CONCEPTUAL}


Desde el ámbito de los servicios, Leonard Berry (1982) recomendó a la industria bancaria estadounidense adoptar un enfoque relacional en sus estrategias y sugirió que ese Marketing debía centrarse en "las relaciones con los clientes" (Berry, 1982:6). Berry reforzó esta teoría del enfoque relacional en una conferencia sobre Marketing de Servicios en la "American Marketing Association" en donde se utilizó por primera vez el término de Marketing de Relaciones y la definió en el sentido de "atraer, mantener y mejorar las relaciones que se mantienen con el cliente" (Berry, 1983:25). A partir de esta definición (Berry, 1983), se empezó a desarrollar una nueva línea de pensamiento más relacional, la cual venía a probar que centrarse exclusivamente en la transacción podía llevar a ignorar gran parte de la esencia del concepto de Marketing. Esto fue lo que indujo a Hunt a entender el Marketing como "la ciencia del comportamiento que busca explicar las relaciones de intercambio" (1983:9-17)

Desde el contexto industrial, Lewit argumentó que este nuevo pensamiento relacional suponía "una ampliación del alcance del Marketing, pasando de las transacciones individuales a las relaciones consumidorproductor".

En 1985, la "American Marketing Association" (AMA) definió el Marketing como "el proceso de planificar y ejecutar la concepción del producto, precio, promoción y distribución de ideas, bienes y servicios, para crear intercambios que satisfagan tanto objetivos individuales como de las organizaciones" (Nova, 2005: 26). Según esta definición de la $A M A$, se entendió que el objetivo principal del Marketing era conseguir un elevado nivel de satisfacción para todas las partes involucradas en la relación. Sin embargo, numerosos 
académicos europeos la criticaron por entender que solo era adecuada para cierto tipo de mercados (Gummeson, 1987).

En 1990, desde la línea de pensamiento nórdica, Grönroos definió el Marketing de Relaciones como el proceso de "establecer, mantener y mejorar las relaciones con los clientes y otros socios o partes del intercambio para que los objetivos de las partes involucradas se cumplan. Esto se lograría mediante un intercambio mutuo y el cumplimiento de las promesas" (Grönroos 1990:5). Tal definición respondía a la necesidad de entender el Marketing de Relaciones desde una perspectiva más amplia, incorporando todo tipo de actores (proveedores, distribuidores, empleados) implicados en las relaciones de la organización. Posteriormente, Grönroos matizó que estas relaciones con los actores tenían que ser frecuentes, pero no necesariamente asiduas.

Otra de las aportaciones que, desde una perspectiva más amplia, ha gozado de gran aceptación entre los académicos fue la realizada por Christopher et al. (1991), quienes describieron el Marketing de Relaciones como "la síntesis del servicio al cliente, la lealtad y el marketing" (Christopher, et al., 1991:5). Para estos autores, el Marketing de Relaciones surgió de la necesidad de integrar aspectos como el marketing, el servicio al cliente y la calidad con el fin de aportar a los clientes de los mercados seleccionados por la empresa un valor único que podía ser mantenido a lo largo del tiempo. Por ello, resaltan la importancia que tiene la correcta coordinación de las relaciones de una empresa, dado que las relaciones externas están en correlación con la calidad de las relaciones internas.

En la misma línea indicada por Grönroos (1990), surgieron otras dos nuevas definiciones; en la una, desde una perspectiva más amplia, Morgan y Hunt (1994) propusieron una definición que les llevó a sostener que el 
Marketing de Relaciones se refiere a "todas las actividades de marketing dirigidas al establecimiento, desarrollo y mantenimiento de intercambios relacionales exitosos" (Morgan y Hunt 1994:22). En la otra, Sheth y Parvatiyar (1994) afirmaron que las definiciones de Berry (1983), de Grönroos (1990), y de Morgan y Hunt (1994) incluían aspectos del proceso de desarrollo y del mantenimiento de las relaciones, pero habría que añadir las relaciones de cooperación y de colaboración entre la empresa y los clientes y catalogar los clientes como uno o como muchos, incluidos los consumidores finales, distribuidores o miembros del canal y los clientes de empresa a empresa. Estas afirmaciones les llevaron a entender el Marketing de Relaciones como "la comprensión, explicación y gestión permanente de las relaciones comerciales de colaboración entre proveedores y clientes" (Morgan y Hunt, 1994:2).

Como podemos constatar, los conceptos e ideas anteriormente mencionados estaban presentes en la nueva definición que planteó Grönroos (1994) en la que se afirmaba que por Marketing de Relaciones él entendía el proceso de "identificar, establecer, mantener, intensificar y, cuando sea necesario, terminar las relaciones con los clientes y otras partes interesadas, con el fin de obtener un beneficio para todas las partes implicadas. Esto se puede lograr mediante un intercambio mutuo y por el cumplimiento de las promesas" (Grönroos, 1994:9).

Siguiendo la idea de Grönroos, posteriormente Gummeson (1997) realizó un esfuerzo por sintetizar las características principales del Marketing de Relaciones, que resumimos en estos términos: (1) representa la síntesis de las relaciones, redes e interacciones; (2) aporta valor para todas las partes involucradas, creado a través de un proceso de interacción entre clientes, proveedores, empleados, competidores y otros. Los proveedores y clientes son co-productores, ya que crean valor para los demás; (3) son valores: "más 
ganar-ganar y menos ganar-perder". Es decir, todos somos iguales en la relación y todas las partes tienen una responsabilidad. Para ello, es necesario estar activos y desarrollar relaciones a largo plazo; (4) son aspectos operativos. Es necesario saber y conocer todas las relaciones posibles de una organización para poder desarrollar cualquier tipo de estrategia; (5) es cartera de relaciones. La selección de las relaciones es parte del proceso de planificación de marketing; (6) el Marketing de Relaciones es una síntesis del marketing-mix, de la gestión del Marketing Tradicional, del Marketing de Servicios, de la red del Marketing Industrial, de la Teoría de las Organizaciones y de otras observaciones profesionales; (7) el Marketing de Relaciones es una orientación, un aspecto de la gestión total de la empresa que no puede afectar solo al departamento de Marketing; (8) el Marketing de Relaciones ha de manifestarse como una organización imaginaria (virtual, en red); (9) el Marketing de Relaciones retiene a los clientes y desarrolla la duración de dichas relaciones. Esto se traduce en una mayor rentabilidad y estabilidad para la organización; (10) el Marketing de Relaciones añade colaboración entre competidores, reguladores e instituciones. Esta simbiosis entre estas tres fuerzas contribuye a un equilibrio de marketing y a una óptima economía de mercado; (11) el Marketing de Relaciones es el marketing de la nueva economía. Es vista como sociedad de servicios, la sociedad de la información, la sociedad del valor (a través de un trato más personalizado); (12) el Marketing de Relaciones es la base para un marketing más realista que está ya presente en la educación del marketing. En la práctica, los negocios se llevan a cabo principalmente a través de relaciones; (13) el Marketing de Relaciones se puede aplicar a cualquier contexto u organización, pero se debe analizar la cartera de relaciones para cada situación.

Para Gummesson (1999:1), el Marketing de Relaciones es un concepto que se desarrolla lejos del "dialogo de doble vía" entre el proveedor y el 
consumidor (Egan, 2011:37). Las relaciones van desde la simple diada hasta las redes más complejas donde las partes entran en un contacto activo, denominándolo red de redes.

Jeringón (2002) manifestó que las definiciones de Grönroos (1990), de Morgan y Hunt (1994) y de Gummesson (1996) podían ser consideradas como las definiciones más acertadas. Asimismo, Harker (1999:15), basándose en siete categorías del Marketing de Relaciones (nacimiento, desarrollo, mantenimiento, interacción temporal, salidas y el contenido emocional), "llegó a la conclusión de que la definición presentada por Grönroos" (1994) era la mejor y más aquilatada, puesto que cubría la conceptualización fundamental del Marketing de Relaciones y que había sido aceptada por la mayoría de los académicos internacionales de esta disciplina.

Kotler (2003:85) afirma que una de las cosas que más valor aporta a una organización son las relaciones que se establecen con los consumidores, empleados, proveedores, distribuidores, mayoristas y minoristas. Kotler entiende el Marketing de Relaciones como "un cambio de paradigma. De pensar solamente en términos competitivos y de conflicto, se pasa a pensar en términos de interdependencia mutua y de cooperación". $Y$ resume sus principales características en las siguientes: (1) se fija antes en los socios y los clientes que en los productos de la empresa; (2) pone más énfasis en la fidelización de los clientes y en el crecimiento que en las nuevas adquisiciones de clientes; (3) se basa más en equipos multifuncionales que en el trabajo de departamentos aislados; (4) se centra más en escuchar y aprender que en hablar.

El pionero en la introducción del Marketing de Relaciones en España, Alet (1994), puso énfasis en su definición en el establecimiento y en 
eldesarrollo de las relaciones con todos los interlocutores de la relación. Para ello, definió el Marketing de Relaciones como "el proceso social y directivo de establecer y cultivar relaciones con los clientes, creando vínculos con beneficios para cada una de las partes, incluyendo a vendedores, prescriptores, distribuidores y cada uno de los interlocutores fundamentales para el mantenimiento y explotación de la relación" (1994:189).

Reinares y Ponzoa destacan la importancia de crear vínculos estables y diferenciadores con los mejores clientes. En consecuencia, por Marketing de Relaciones entienden "las diferentes acciones e iniciativas desarrolladas por una empresa hacia sus diferentes públicos o hacia un determinado público o segmento de los mismos, dirigidas a conseguir su satisfacción en el tiempo, mediante la oferta de servicios y productos ajustados a sus necesidades y expectativas, incluida la creación de canales de relación estables de intercambio de comunicación y valor añadido, con el objeto de garantizar un clima de confianza, aceptación y aportación de ventajas competitivas que impida la fuga hacia otros competidores"(Reinares y Ponzoa, 2006:19).

La "American Marketing Association" en sus dos últimas propuestas ha entendido el Marketing como "una función de la organización y un conjunto de procesos dirigidos a crear, comunicar y distribuir valor a los clientes y a gestionar las relaciones con los clientes de forma que beneficie a la organización y a sus públicos de interés" (AMA, 2004); más recientemente, lo ha definido como "una actividad de la organización y un conjunto de procesos para crear, comunicar, distribuir e intercambiar ofertas que tengan valor para los consumidores, clientes, socios y sociedad en general" (AMA,2007). 


\begin{tabular}{|c|c|}
\hline $\begin{array}{c}\text { Y } \\
\text { REFERENCIA }\end{array}$ & DEFINICIONES \\
\hline $\begin{array}{l}\text { Hammarkvist et al. } \\
\text { (1982) en Agariya y } \\
\text { Singh (2011:207) }\end{array}$ & $\begin{array}{l}\text { "Comprende todas las actividades de la empresa para construir, mantener y } \\
\text { desarrollar relaciones con los clientes". }\end{array}$ \\
\hline $\begin{array}{l}\text { Berry } \\
(1983: 25)\end{array}$ & $\begin{array}{l}\text { "Atraer, mantener y -en múltiples organizaciones de servicios- mejorar las } \\
\text { relaciones que se mantienen con el cliente". }\end{array}$ \\
\hline $\begin{array}{l}\text { Levitt } \\
\text { (1983) en Agariya y } \\
\text { Singh (2011:207) }\end{array}$ & $\begin{array}{l}\text { "Es un proceso que consiste en cinco etapas, a saber, la conciencia, la } \\
\text { exploración, la expansión, el compromiso y de disolución". }\end{array}$ \\
\hline $\begin{array}{l}\text { Hallen y } \\
\text { Wiedersheim-Paul } \\
\text { (1984) en Agariya y } \\
\text { Singh (2011:207) }\end{array}$ & $\begin{array}{l}\text { "Es un proceso que consta de cuatro etapas, a saber, pre-contacto, la } \\
\text { interacción inicial, el desarrollo y la relación madura". }\end{array}$ \\
\hline $\begin{array}{l}\text { Jackson } \\
(1985: 2)\end{array}$ & $\begin{array}{l}\text { "Es el tipo de marketing orientado hacia relaciones sólidas y duraderas con los } \\
\text { clientes individualmente considerados". }\end{array}$ \\
\hline $\begin{array}{l}\text { Berry y Gresham } \\
(1986: 43)\end{array}$ & "Atraer, retener e intensificar las relaciones con el cliente". \\
\hline $\begin{array}{l}\text { Paul } \\
(1988: 21)\end{array}$ & $\begin{array}{l}\text { "Es la identificación y cultivo de un alto potencial, prospectos y clientes a largo } \\
\text { plazo". }\end{array}$ \\
\hline $\begin{array}{l}\text { Turnbull y Wilson } \\
\text { (1989) en Agariya y } \\
\text { Singh (2011:207) }\end{array}$ & $\begin{array}{l}\text { "Es la formación de relaciones entre compradores y vendedores a largo plazo } \\
\text { a través de la creación de vínculos estructurales y sociales entre las } \\
\text { empresas". }\end{array}$ \\
\hline $\begin{array}{l}\text { Grönroos } \\
(1990: 138)\end{array}$ & $\begin{array}{l}\text { "Establecer, mantener y mejorar las relaciones con los clientes y otras partes } \\
\text { interesadas con el fin de obtener un beneficio para todas las partes implicadas. } \\
\text { Esto se puede lograr mediante un intercambio mutuo y por el cumplimiento de } \\
\text { las promesas". }\end{array}$ \\
\hline $\begin{array}{l}\text { Rapp y Collins } \\
\text { (1990) en Agariya y } \\
\text { Singh (2011:207) }\end{array}$ & $\begin{array}{l}\text { "Es crear y mantener relaciones duraderas entre la empresa y sus clientes que } \\
\text { sean beneficiosas para ambas partes". }\end{array}$ \\
\hline $\begin{array}{l}\text { Gummesson } \\
\text { (1990) en Harker } \\
(1999: 18)\end{array}$ & $\begin{array}{l}\text { "Es la construcción, el mantenimiento y la liquidación de las redes y las } \\
\text { relaciones interactivas entre el proveedor y el cliente, a menudo con } \\
\text { consecuencias a largo plazo". }\end{array}$ \\
\hline $\begin{array}{l}\text { Kotler } \\
(1991: 4-5)\end{array}$ & $\begin{array}{l}\text { "Es la tarea empresarial de establecer, mantener y potenciar las relaciones } \\
\text { con los clientes y otros participantes, socios en el beneficio, para que se } \\
\text { cumplan los objetivos de todos los implicados". }\end{array}$ \\
\hline $\begin{array}{l}\text { Berry y Parasuraman } \\
(1991: 133)\end{array}$ & "Es el proceso de atraer, desarrollar y conservar las relaciones con el cliente". \\
\hline $\begin{array}{l}\text { Christopher et al. } \\
(1991: 5)\end{array}$ & "Es la síntesis del servicio al cliente, la fidelidad y el marketing". \\
\hline $\begin{array}{l}\text { Pathmarajah } \\
\text { (1991) en Agariya y } \\
\text { Singh (2011:207) }\end{array}$ & $\begin{array}{l}\text { "Es el proceso por el cual el vendedor y el comprador se unen en una sólida } \\
\text { relación personal, profesional y mutuamente rentable en el tiempo". }\end{array}$ \\
\hline
\end{tabular}

REDMARKA UIMA-Universidad de A Coruña - CIECID

Año IX, Número 16, (2016), v I pp. 41- 66

http://www.redmarka.net/ ISSN 1852-2300 
Shani y Chalasani (1992:34)
"Es un esfuerzo integrado por identificar, mantener y construir una red global con clientes individuales, reforzando permanentemente dicha red para el beneficio mutuo de ambas partes por medio de contactos interactivos, individualizados y de amplio valor añadido sobre un período extenso de tiempo".

Tabla 2.2: Resumen de las definiciones de Marketing de Relaciones (1982-2013)

(Continuación en la siguiente página)
$Y$

REFERENCIA

Webster

(1992) en Agariya y

Singh (2011:207)

Doyle y Roth

(1992:59)

Porter

(1993:14)

Cram

(1994:19)

Evans y Laskin

(1994:440)

Barney y Hansen (1994) en Agariya y

Singh

(2011:207)

Morgan y Hunt (1994:22)

Cravens y Piercy

(1994)

Doyle

(1994:36)

Matthyssens y Van den Bulte (1994) en Agariya y Singh (2011:207)

Grönroos

(1994:9)

Gummesson

(1994) en Harker (1999:18)
"Se trata de un proceso de comprensión y gestión de relaciones rentables con los clientes mediante la entrega de un valor superior al cliente, servicio y satisfacción".

"Es ganar la posición del proveedor mediante el desarrollo de la confianza en las cuentas clave durante un período de tiempo".

"Es el proceso mediante el cual ambas partes, vendedor-comprador, establecen una relación efectiva, eficiente, agradable, entusiasta y ética, la cual es personalmente, profesionalmente y rentablemente valiosa para ambas partes".

"La aplicación coherente del conocimiento actualizado a diario acerca de los clientes individuales para el diseño del producto/servicio, lo que será comunicado de manera interactiva con la finalidad de desarrollar una relación continua y de largo plazo que sea mutuamente beneficiosa".

"Enfoque centrado en el cliente por el cual una compañía busca relaciones comerciales a largo plazo con clientes actuales y potenciales".

"Es la actividad en la que el desarrollo de rutinas de comportamiento prescriptivo se ha relacionado con la disminución de los costos de transacción resultantes de la negociación y el seguimiento, el aumento de la innovación, la creación de ventaja posicional y mejora del desempeño".

"Todas las actividades de marketing dirigidas al establecimiento, desarrollo y mantenimiento de intercambios relacionales exitosos".

"Es la comprensión, explicación y gestión de las relaciones de colaboración continuas en los negocios entre los proveedores y sus clientes".

"Son una serie de transacciones entre las partes a largo plazo".

"Se trata de la construcción, el apoyo y ampliación de relaciones con los clientes".

"Identificar, establecer, mantener, intensificar y, cuando sea necesario, terminar las relaciones con los clientes y otras partes interesadas con el fin de obtener un beneficio para todas las partes implicadas. Esto se puede lograr mediante un intercambio mutuo y por el cumplimiento de las promesas".

"Es poner énfasis en una relación interactiva a largo plazo entre el proveedor y el cliente, y una rentabilidad a largo plazo".

REDMARKA UIMA-Universidad de A Coruña - CIECID

Año IX, Número 16, (2016), v I pp. 41- 66

http://www.redmarka.net/ ISSN 1852-2300 


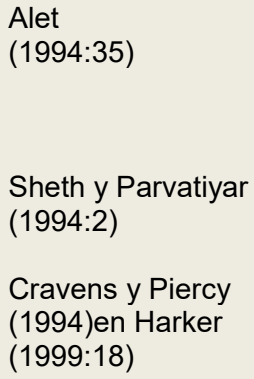

"Es el proceso social y directivo de establecer y cultivar relaciones con los clientes, creando vínculos con beneficios para cada una de las partes, que incluyen a vendedores, prescriptores, distribuidores y a cada uno de los interlocutores fundamentales para el mantenimiento y explotación de la relación".

"La comprensión, explicación y gestión permanente de las relaciones comerciales de colaboración entre proveedores y clientes".

"Es la comprensión, explicación y gestión de las relaciones de colaboración continuas en los negocios entre los proveedores y sus clientes".

Tabla 2.2: Resumen de las definiciones de Marketing de Relaciones (1982-2013)

(Continuación en la siguiente página) AUTORES

$Y$

\section{REFERENCIA}

Håkansson y

Snehota (1995:25)

Möller y Wilson

(1995:1)

Perrien, y Ricard

(1995:38)

Stone, Woodcock y

Wilson

(1996:675)

Paravatiyar (1996)

en Harker (1999:18)

Ravald y Grönroos (1996) en Harker

(1999:18)

Takala y Uusitalo (1996) en Harker (1999:18)

Tzokas y Saren (1996) en Harker (1999:18)

Buttle (1996) en Harker
"Es la conexión de actividades, los vínculos personales y los lazos entre los recursos de las partes de intercambio".

"Es la comprensión, creación y gestión de las relaciones de intercambio entre los agentes económicos, los fabricantes, los proveedores de servicios, miembros del canal y los consumidores finales".

"Es un proceso de marketing asimétrico y personalizado. Este proceso se desarrolla a lo largo del tiempo para obtener beneficios bilaterales y consta de unos conocimientos profundos sobre las características y necesidades de los clientes".

"Es el uso de un amplio espectro de instrumentos de marketing con el fin de identificar personalmente a los clientes de una empresa, de crear una relación entre la empresa y sus clientes (relación que se verá fortalecida a través de las diversas transacciones) y de dirigir las relaciones en el beneficio de los clientes y de la empresa".

"Es el proceso de cooperación con los clientes para mejorar la productividad del marketing a través de la eficiencia y la eficacia".

"El núcleo del marketing de relaciones son las relaciones, el mantenimiento de las relaciones entre la empresa y los actores en su micro-ambiente... La idea es, ante todo, conseguir la lealtad del cliente para crear una relación estable y mutuamente beneficiosa a lo largo del tiempo".

"Es establecer, fortalecer y desarrollar relaciones con los clientes. Se basa en establecer relaciones rentables con los clientes y la búsqueda de objetivos individuales y organizacionales a lo largo del tiempo"

"Es el proceso de planificación, desarrollo y fomento de un clima de relación que promueva el dialogo entre una empresa y sus clientes con el objetivo de inculcar la comprensión, la confianza, el respeto de las capacidades de los demás y preocupaciones".

"Es el desarrollo y el mantenimiento de las relaciones mutuamente beneficiosas con los mercados estratégicamente significativos".

REDMARKA UIMA-Universidad de A Coruña - CIECID

Año IX, Número 16, (2016), v I pp. 41- 66

http://www.redmarka.net/ ISSN 1852-2300 


\begin{abstract}
$(1999: 18)$
Bennett

(1996:420)

Anton

(1996) en Agariya y

Singh(2011:208)

Jolson

(1997) en Agariya y

Singh(2011:209)

Bendapudi y Berry

(1997) en Agariya y

Singh(2011:209)

Murphy, Stevens y

Macleod (1997:44)

"Es el desarrollo organizacional y mantenimiento de mutuas relaciones cordiales con los clientes por la vía de una integración total de la información y de los sistemas de gestión de la calidad, soporte de servicio, estrategia de negocio y misión organizacional con el objeto de satisfacer al cliente y asegurar una prolongación rentable del negocio".

"Es una estrategia de marketing que integra tecnología, proceso y todas las actividades de negocio que rodean al cliente".

"Es un enfoque estratégico desarrollado por un proveedor dispuesto a establecer relaciones a largo plazo y mutuamente beneficiosas para ambas partes".

"Se centra en comprender y en satisfacer las necesidades del cliente".

"Es la construcción y permanente cultivo de relaciones de intercambio mutuamente beneficiosas a largo plazo que una organización realiza con sus clientes y con otros grupos de interés".
\end{abstract}

Tabla 2.2: Resumen de las definiciones de Marketing de Relaciones (1982-2013)

(Continuación en la siguiente página) AUTORES REFERENCIA

Ballantyne

(1997) en Harker

(1999:18)

O'Malley et al. (1997) en Harker (1999:18)

Coviello et al. (1997:23)

Achrol

(1997) en

Hefferman

(2004:115)

Gummesson

(1997:32)

Morris et al. (1998) en Agariya y Singh (2011:209)

Srivastava et al.
"Es un marco de disciplina emergente para crear, desarrollar y mantener el intercambio de valor entre las partes involucradas mediante el cual las relaciones de intercambio evolucionan para proporcionar enlaces continuos y estables en la cadena de suministro".

"Es la identificación, especificación, iniciación, mantenimiento y (cuando sea oportuno) disolución de las relaciones a largo plazo con los clientes clave y con otras partes, a través del mutuo intercambio, el cumplimiento de las promesas y la adhesión a las normas de las relaciones para satisfacer los objetivos y mejorar la experiencia de las partes implicadas".

"Es una actividad integradora que implica funciones a través de la organización, poniendo énfasis en la facilitación, la creación y el mantenimiento de la relación en el tiempo".

"Es la construcción de relaciones duraderas basadas en la estructura de los beneficios a largo plazo y la afinidad mutua entre el comprador y el vendedor".

"Se trata del marketing visto como un conjunto de relaciones, redes e interacciones".

"Es una orientación estratégica adoptada por el comprador y el vendedor de la organización, lo que representa un compromiso a largo plazo y la colaboración para un beneficio mutuo".

"Abarca todos los aspectos de la identificación de los clientes, la creación de

REDMARKA UIMA-Universidad de A Coruña - CIECID

Año IX, Número 16, (2016), v I pp. 41- 66

http://www.redmarka.net/ ISSN 1852-2300 


\begin{abstract}
(1998) en Agariya y Singh (2011:209)

conocimiento de los clientes, la creación de valores de los clientes y dar forma a las percepciones de los clientes de una organización y sus productos".

Reinares y Calvo (1999) en Reinares y Ponzoa (2006:19)

Harker (1999:16)

Gordon

(1999:1)

"Parte de la premisa de que toda actividad comercial es como la vida misma: se basa en las relaciones y, para ello, utiliza todas las técnicas a su alcance, incluyendo las últimas tecnologías en comunicación y producción para convertir cualquier contacto con un cliente real o potencial en una relación duradera y satisfactoria para la marca y el consumidor".

"Es el proceso de identificar y establecer, mantener y mejorar y, cuando sea necesario, terminar las relaciones con los clientes ( $y$ otras partes) de manera que se cumplan los objetivos de todas las partes con respecto a las variables económicas y de otro tipo. Esto se puede lograr a través de una decisión mutua y el cumplimiento de las promesas".

"Es el proceso continuo de identificar y crear un nuevo valor con los clientes individuales y luego compartir los beneficios con esa asociación. Se trata de comprender y gestionar el enfoque de colaboración entre proveedores y clientes para la creación de un valor mutuo, el intercambio a través de la interdependencia y la alineación de la organización".
\end{abstract}

Galbreath y Rogers (1999) en Agariya y Singh (2011:209)

Weitz y Bradford (1999) en Hefferman $(2004: 115)$

"Se trata de la gestión de la tecnología, los procesos, los recursos de información y las personas necesarias para crear un entorno que permita a las empresas tener una visión de 360 grados de sus clientes".

"Se refiere a una serie de actividades de marketing dirigidas hacia el desarrollo de asociaciones fuertes en las relaciones business-to-business que serán clave para el cliente".

Tabla 2.2: Resumen de las definiciones de Marketing de Relaciones (1982-2013)

(Continuación en la siguiente página) AUTORES

REFERENCIA

Santesmases et al. (2001)

Parra y Molinillo (2001:36)

Reinares y Ponzoa (2002:19)
DEFINICIONES

"Es el establecimiento de relaciones estables y duraderas con los clientes, que permitan de un modo más efectivo su satisfacción y lealtad. Todo ello con la ayuda de la tecnología y bases de datos relacionales".

"Es establecer, desarrollar y comercializar relaciones a largo plazo, medio y corto plazo con clientes, porveedores, distribuidores, entidades laterales (competidores, organizaciones no lucrativas y gobierno) y ámbito interno (empleados, departamentos funcionales y unidades de negocio), tales que permitan el mayor valor ofertado al consumidor final y, a la vez, la consecución de los objetivos de todas las partes mencionadas de la forma más eficiente para cada una de ellas".

"Las diferentes acciones e iniciativas desarrolladas por una empresa hacia sus diferentes públicos o hacia un determinado público o segmento de ellos mismos, dirigidas a conseguir su satisfacción en el tiempo mediante la oferta de servicios y productos ajustados a sus necesidades y expectativas, lo que incluye la creación de canales de relación estables de intercambio de comunicación y valor añadid para garantizar un clima de confianza, aceptación y aportación de ventajas competitivas que impida la fuga hacia otros competidores".

REDMARKA UIMA-Universidad de A Coruña - CIECID

Año IX, Número 16, (2016), v I pp. 41- 66

http://www.redmarka.net/ ISSN 1852-2300 


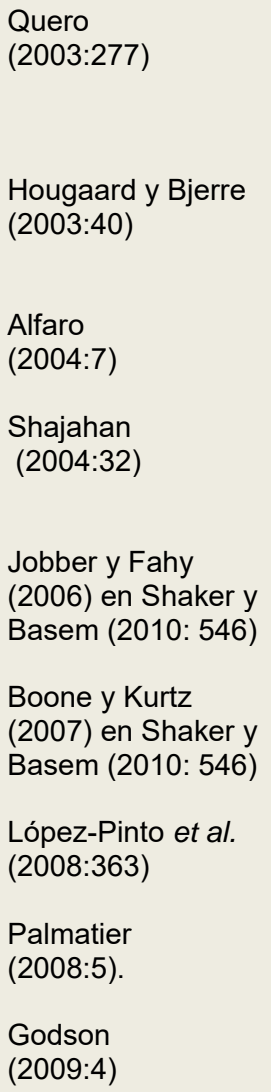

"Es el proceso de identificar y planificar relaciones con clientes y otros agentes implicados con el fin de conseguir el cumplimiento de los objetivos de todas las partes involucradas a través del desarrollo de relaciones beneficiosas para todos".

"Es el comportamiento que una empresa tiene como propósito establecer, mantener y desarrollar con los clientes relaciones rentables y competitivas en beneficio de ambas partes".

"Es la gestión estratégica de relaciones de colaboración con clientes y otros actores con el objetivo de crear y distribuir valor de forma equitativa".

"Es el proceso de participación en actividades de colaboración, cooperación y programas con los usuarios finales con el objetivo de crear y aumentar el valor mutuo y reducir los costes económicos".

"Es el proceso de creación, desarrollo y mejora de la relación con los clientes y otras partes interesadas".

"Se refiere al desarrollo, crecimiento y mantenimiento de la relación a largo plazo con los distintos clientes, proveedores, empleados y otros socios que sea rentable y beneficioso para ambas partes".

"Es el proceso de administrar las relaciones de la empresa de una manera rentable".

"Es el proceso de identificar, desarrollar, mantener y finalizar el intercambio relacional con el fin de mejorar el rendimiento".

"Es la relación entre todos los actores implicados y que sea mutuamente beneficiosa para todas las partes implicadas".

Fuente: Elaboración propia

El "Chartered Institute of Marketing" (CIM, Egan, 2011) entiende por Marketing "el proceso de gestión responsable de identificar, anticipar y satisfacer las necesidades del cliente rentable". Esta definición aporta a la discusión una serie de supuestos que resultan importantes para el desarrollo de la estrategia relacional. Pues, el "proceso" asume que el Marketing Tradicional se basa en una serie de actividades llevadas a cabo en el marco del "Marketing mix" y también sugiere implícitamente la idea de "identificar, anticiparse y satisfacer a los clientes"; actividades que son rentables en el sentido de que a la organización compete la responsabilidad de llevar a cabo esta relación de una manera competitiva (Gordon, 1998). Téngase en cuenta 
que el Marketing Tradicional, por encima de todo, "no reconocía el valor de los clientes a largo plazo" (Buttle, 1996:2). En este sentido, el mayor desafío para los defensores del Marketing de Relaciones consistía en demostrar que la cooperación mutua, a diferencia de la competencia y el conflicto, conducía a la creación de valor (Sheth y Parvatiyar, 1995:399).

\section{RESULTADOS Y CONCLUSIONES}

En este apartado, recogemos las principales conclusiones que se derivan de la investigación desarrollada. Respondiendo al único objetivo planteado al principio, nuestra de investigación nos permite afirmar que el Marketing de Relaciones es: (1) un proceso que puede y debe aplicarse a cualquier tipo de sector; (2) un proceso constituido por las fases de identificar, establecer, desarrollar, mantener y si es necesario terminar la relación; (3) un proceso que implica relaciones de cinco tipos, que pueden ser relaciones con los empleados, relaciones con los clientes, relaciones verticales, relaciones horizontales y relaciones mediadas por computadoras (virtuales); (4) un proceso que lleva a la consecución de beneficios mutuos a través de la cocreación de valor para ambas partes; (5) un proceso en el que la relación ha de ser de calidad, calidad que vendrá determinada por la satisfacción, por la confianza y por el compromiso de ambas partes; (6) un proceso en el que el objetivo principal se cifrará en la fidelidad y en el cumplimiento de las promesas. En este sentido, los diez conceptos que más se han utilizado para definir el Marketing de Relaciones han sido la confianza, la satisfacción, la experiencia, el compromiso, la fidelidad, la comunicación, la calidad de servicio, la cocreación del valor, la tecnología y la retención de los clientes. Por tanto, en coherencia con lo que venimos analizando, podemos expresar nuestra coincidencia con Harker en que la definición mejor y más adecuada de Marketing de Relaciones es la proporcionada por Grönroos (1994), de la cual 
partimos y que reformulamos, a modo de contribución, en los términos siguientes: "el Marketing de Relaciones es el proceso de identificar, establecer, desarrollar, mantener y, cuando sea necesario, terminar las relaciones entre la organización, los clientes y otros socios a lo largo del tiempo con el fin de satisfacer beneficios mutuos y cocrear valores mediante interacción".

Aunque no entraba en el objetivo inicial sobre el concepto de Marketing de Relaciones, también es importante añadir la conclusión a que hemos llegado desde una perspectiva nominal. En numerosos libros y artículos de investigación, aparece dicho concepto denotado como "Marketing Relacional", lo que nos llevó a probar la falta de rigor nominal a la hora de utilizar dicho término. Tras haber realizado un análisis nominal y conceptual, podemos sostener que el término más adecuado para traducir la expresión inglesa "Relationship Marketing" al español es el de Marketing de Relación o de Relaciones. El término inglés "relation" es un sustantivo abstracto cuyo significado es "the way in which two or more concepts, objects, or people are connected; a thing's effect on or relevance to another (= la manera en la que dos o más conceptos, objetos o personas).

La proliferación de definiciones tan diferentes desde 1982 hasta 2013 refleja la evolución que en tanto concepto ha experimentado el Marketing de Relaciones. Tales definiciones pueden ser tomadas como la expresión del contexto en el que se han realizado y también del debate existente durante años entre académicos y profesionales sobre qué es realmente el Marketing de Relaciones y sobre cuáles fueron y cuáles son sus objetivos (Harwood y Garry, 2006). A pesar de ello, todas las definiciones recopiladas comparten numerosos elementos, por lo que resultan contradictorias entre sí. Uno de esos elementos comunes está en que todas conciben la promoción de las relaciones como factor esencial dentro y fuera de la organización. Por ello, casi todas 
resaltan la necesidad de desarrollar relaciones a largo plazo con todos los clientes, competidores, proveedores y empleados. Pues, de acuerdo con la escuela nórdica (Grönroos, 1994; Gummeson, 1996) y con El Grupo IMP (Hakansson 1982, Ford 1990), para ser un competidor efectivo (en la economía global), se requiere ser un cooperador efectivo (en alguna red).

En este sentido, el Marketing de Relaciones concibe el entorno empresarial como amplio, entendiendo por tal las relaciones existentes entre la empresa y los otros actores que la rodean (proveedores, agentes de interés, empleados, administración, etc.). Estas distintas relaciones se logran mediante el reconocimiento de unas fases o estadios sucesivos dentro del proceso en el que aparecen acuñadas bajo los términos de identificar, establecer, desarrollar, mantener y terminar cuando sea necesario. A lo largo de dicho proceso, la relación puede medirse por la calidad del servicio, el valor de la relación, la confianza, la satisfacción, el compromiso, la fidelidad, conceptos que son todos esenciales para que la relación entendida como proceso se mantenga a lo largo del tiempo y, en consecuencia, se alcance un beneficio mutuo, la compra de repetición, comunicación "boca-oído", la retención de los clientes, el ganar clientes nuevos y la recuperación de clientes perdidos. Por tanto, en coherencia con lo que venimos analizando, podemos expresar nuestra coincidencia con Harker en que la definición mejor y más adecuada de Marketing de Relaciones es la proporcionada por Grönroos (1994) de la cual partimos para formular a modo de contribución la nuestra en los términos siguientes:

"Marketing de Relaciones es el proceso de identificar, establecer, desarrollar, mantener $\mathrm{y}$, cuando sea necesario, terminar las relaciones entre la organización, los clientes y otros socios a lo largo del tiempo con el fin de satisfacer beneficios mutuos y cocrear valores mediante la interacción" (Sarmiento, 2014). 


\section{BIBLIOGRAFÍA}

Achrol, R. S. (1997): "Changes in the theory of Interorganizational Relations in Marketing; Toward a Network Paradigm", Journal of the Academy of Marketing Science, 25 (1), 56-71.

Agariya A. y Singh, D. (2011): "What Really Defines Relationship Marketing? A Review of Definitions, General and Sector-specific Defining Constructs", Journal of Relationship Marketing, 10 (4), 203-237

Alet, J. (1994): Marketing Relacional, Ed. Gestion 2000.com, Barcelona.

Alfaro Faus, M. (2004): Temas clave de Marketing Relacional. Espana: Mc Graw- Hill.

AMA (1985): "AMA Board Aprovess New Marketing Definition", Marketing News, 19, 5, 1.

Barroso, C. y Martín, E. (1999): Marketing Relacional, Madrid: ESIC.

Bennett, R. (1996): "Relationship Formation and Governance in Consumer Markets: Transactional Analysis versus the Behavioural Approach", Journal of Marketing Management, 12 (5), 417-436.

Berry, L. L. (1983): "Relationship Marketing”, L. Berry, G. L Shostack and G. D Upah, (eds.): Emerging Perspectives on Services Marketing, Chicago American Marketing Association, 25-28.

Berry, L. L. y L. G. Gresham (1986): "Relationship Retailing: Transforming Customers into Clients", Business Horizons, 29 (Nov./December), 43-47.

Berry, L. L. y Parasuraman, A. (1991): Marketing Services: Competing through Quality, New York: Free Press.

Buttle, F. (editor) (1996): Relationship Marketing: Theory and Practice, London: Paul Chapman Publishing.

Christopher, M., Payne, A. y Ballantyne, D. (1991), Relationship Marketing: Bringing Quality, Customer Service and Marketing Together, Oxford: Butterworth-Heinemann.

Coviello, N. E., Brodie, R. J. and Munro, H. J. (1997):“Understanding contemporary marketing: Development of a classification scheme", Journal of Marketing Management, 13 (6), 501-522.

Cram, T. (1994), The Power of Relationship Marketing: How to Keep Customers for Life, London: Pitman Publishing.

Cravens, D. W., y Piercy, N. E. (1994): "Relationship Marketing and Collaborative Networks in Service Organization", International Journal of Service Industry Management, 5 (5), 39-53.

Dann, S., y Dann, S. (2000): Strategic Internet Marketing, Milton, John Wiley and Sons Australia

De la Antonia, D. (2009): Hacia el liderazgo europeo en las escuelas de pensamiento de Marketing, Madrid: Vision.

REDMARKA UIMA-Universidad de A Coruña - CIECID

Año IX, Número 16, (2016), v I pp. 41- 66

http://www.redmarka.net/ ISSN 1852-2300 
Doyle, P. (1994): Marketing Management and Strategy, London: Prentice Hal Publishing.

Doyle, S. X., y G. T. Roth (1992), "Selling and Sales Management inAction: The Use of Insight Coaching to Improve Relationship Selling", Journal of Personal Selling and Sales Management, 12 (Winter), 69-74.

Egan, J. (2011): Relationship Marketing: Exploring Relational Strategies in Marketing, Financial Times / Prentice Hall.

Evans, J. R., y Laskin, R. L. (1994): "The relationship Marketing process: A conceptualizationand application", Industrial Marketing Management, 23, 439-452.

Gordon I. (1998): Relationship Marketing, Etobicoke Ontario Canada, John Wiley and Sons.

Grönroos, C. (1990): "Relationship Approach to the Marketing Function in Service Contexts: The Marketing and Organisational Behaviour Interface", Journal of Business Research, 20 (1), 3-12.

Grönroos, C. (1991): "The Marketing Strategy Continuum: Toward a Marketing Concept for the 1990's", Managment Decision, 29 (1) 7-13.

Gummesson, E. (1987): "The New Marketing Developing Long Term InteractiveRelationships", Long Range Planning, 4, 10-20.

Gummesson, E. (1991): "Marketing-Orientation Revisited: The Crucial Role of the Part-Time Marketer", European Journal of Marketing, 2, 60-75.

Gummesson, E. (1994): "Relationship Marketing Operational", International

Gummesson, E. (1996): "Relationship Marketing and Imaginary Organizations: A Synthesis", European Journal of Marketing, 30 (2), 31-44.

Gummesson, E. (1999): Total Relationship Marketing. Ed. Butterworth Heinemann, Oxford.

Håkansson, H. y Snehota, I. (1995): Developing Relationships in Business Networks, Routledge, London.

Harker, M. J. (1999): “Relationship Marketing Defined?”, Marketing Intelligence and Planning, 17 (1),13-21.

Heffernan, T. (2004): Trust formation in cross-cultural business-to-business relationships. Qualitative Market Research: An International Journal, 7(2) 114-125.

Jackson B. B. (1985): "Build Customer Relationships that Last", Harvard Business Review, 63, 120-128.

Journal of Service Industry Management, 5 (5), 5-20.

Kotler P. y Armstrong, G. (2013): Fundamentos de Marketing, Ed. Pearson PrenticeHall, 12a Edicion, Madrid.

Kotler, P. (1992): "Total Marketing", Business Week Advance, Executive Brief, 2.

Küster, I. (2002): La venta relacional, Ed. ESIC, Madrid.

Landeta, J. (1999): El método Delphi. Ariel, Barcelona. 
López-Pinto Ruiz, B., Mas Machuca, M. y Viscarri Colomer, J. (2008): Los pilares del Marketing, Barcelona: Ed. UPC.

Malhotra, N.K. (2013): Investigación de mercados. México: Prentice Hall.

Möller, K. y Wilson, D. (1995): "Interaction and Networks in Perspective", Kristian Moller-David Wilson (Ed.): Business Marketing: An Interaction and Network Perspective, 1-18. Boston: Kluwer Academic Publishers.

Morgan, R. M. y Hunt, S. D. (1994): "The Commitment-Trust Theory of

Mui, L., Mohtashemi, M. y Halberstadt, A. (2002): "A computational model of trust and reputation", Proceedings of the 35th International Conference on System Science, Big Island, Hawaii, 188-197.

Murphy, B., Stevens, D. K., y McLeod, R. (1997): "A Stakeholderism Framework for Measuring Relationship Marketing", Journal of Marketing Theory and Practice, 5 (2), 43-57.

Nevin J. R. (1995), "Relationship Marketing and Distribution Channels: Exploring Fundamental Issues", Journal of the Academy Marketing Sciences, (Fall), 327- 334.

Nova, G. (2005): La gestión de las relaciones con clientes (CRM) como herramienta operativa para fomentar el nivel de lealtad de los turistas que visitan destinos turísticos urbanos, Tesis doctoral, Universidad de Valencia.

O'Malley L., C. Tynan (2000): "Relationship Marketing in Consumer Markets: Rhetoric or reality?", European Journal of Marketing, 34 (7), 797-815.

Oxford Spanish Dictionary (2009): Oxford University Press.

Palmatier, Robert W., (2008): "Interfirm Relational Drivers of Customer Value," Journal of Marketing, 72 (July), 76-89.

Parra, F.; Molinillo, S. (2001): "Marketing relacional y actividad comercial en los centros urbanos", ESIC Market, 109, 33-62.

Paul, T. (1988): "Relationship Marketing for Health Care Providers," Journal of HealthCare Marketing, 8, 20-25.

Payne, A. (2006). Handbook of CRM: Achieving Excellence in Customer Management, Elsevier, Amsterdam.

Payne, A. y P. Frow, (2005): "A strategic framework for customer relationship management", Journal of Marketing, 69 (4), 167-176.

Peterson, R. (1995): "Relationship marketing and consumer", Journal of Academy of Marketing Science, 23 (4), 278-281.

Pine, B. J., Pepper, D., y Rogers, M. (1995): "Do you wantto keep your customers forever?", Harvard Business Review, 73 (2), 103-114.

Quero, M. J. (2003): Marketing Cultural. El enfoque relacional en las entidades escénicas, Ed. Red Espanola de Teatros, Auditorios y Circuitos de Titularidad Pública, Madrid.

Reinares, P. J., y Ponzoa, J. M. (2004): Marketing relacional. Un nuevo enfoque para la seducción y fidelización del cliente, Ed. Finan-cial Times-Prentice Hall, Madrid.

REDMARKA UIMA-Universidad de A Coruña - CIECID

Año IX, Número 16, (2016), v I pp. 41- 66

http://www.redmarka.net/ ISSN 1852-2300 
relationship Marketing", Journal of Marketing, 58, 20-38.

Santesmases Mestre, M., Kosiak, G. y Sánchez F. (2001): Marketing: conceptos y estrategias, Madrid: Piramide.

Sarmiento, J. R. (2014): El Marketing de Relaciones en los medios sociales: estudio empírico de los antecedentes y consecuentes de la calidad de la relación en los sitios web de viajes. Tesis doctoral. Universidad Rey Juan Carlos. Madrid.

Sarmiento, J. R. (2015): Marketing de Relaciones. Aproximación a las relaciones virtuales. Dykinson, Madrid.

Shajahan, S. (2004): Relationship Marketing. New Delhi, India: Tata McGrawHill Publishing Co.

Shaker, T. Ismail y Basem, Y. Alsadi (2010): "Relationship Marketing and Organizational Performance Indicators" European Journal of Social Sciences, 12(4) 545-557.

Shani, D. y Chalasani, S. (1992): "Exploiting Niches Using Relationship Marketing", Journal of Services Marketing, 6 (4), 43-52

Sheth, J. and Parvatiyar, A. (1994): Relationship Marketing: Theory, Methods and Applications, Center for Relationship Marketing, Emory University, Atlanta.

Sheth, J. N. \& Parvatiyar, A. (2000): Handbook of Relationship Marketing, Thousand Oaks, CA: Sage Publications.

Stone, M., Woodcock, N., y Wilson, M. (1996): Managing the change from marketing planning to customer relationship management", Long Range Planning, 29, 675-683.

Vallespín, M. y Molinillo, S. (2014): «El futuro de la intermediación en el sector turístico». Revista de análisis turístico, vol. 17, $\mathrm{n}^{\circ}$ 1, pp. 13-25.

Weitz, B. A and Jap, S. D, (1995): "Relationship Marketing and Distribution Channels", Journal of the Academy of Marketing Science, 23 (4), 305320.

REDMARKA UIMA-Universidad de A Coruña - CIECID

Año IX, Número 16, (2016), v I pp. 41- 66

http://www.redmarka.net/ ISSN 1852-2300 